\title{
Abdominal Pain and Disturbed Bowel Movements are Frequent among Young People. A Population Based Study in Young Participants of the Woodstock Rock Festival in Poland
}

\author{
Ewa Stachowska ${ }^{1}$, Dominika Maciejewska ${ }^{1}$, Karina Ryterska ${ }^{1}$, Piotr Baszuk ${ }^{2}$, Karolina Skonieczna-Żydecka ${ }^{1}$, Maja \\ Czerwińska-Rogowska ${ }^{1}$, Joanna Palma ${ }^{1}$, Anna Gudan ${ }^{1}$, Honorata Mruk ${ }^{1}$, Anna Wolska ${ }^{1}$, Tomasz Mazur ${ }^{1}$, Dagmara \\ Paszkiewicz ${ }^{3}$, Zofia Stachowska ${ }^{4}$, Adam Stachowski ${ }^{5}$, Wojciech Marlicz ${ }^{6}$
}

1) Dept. Biochemistry and

Human Nutrition, Pomeranian

Medical University, Szczecin;

2) Dept. Genetics and

Pathomorphology,

Pomeranian Medical

University, Szczecin;

3) Poznan University of Life

Sciences, Poznań;

4) University of Szczecin,

Szczecin;

5) Poznan University of

Medical Science, Poznań;

6) Dept. Gastroenterology,

Pomeranian Medical

University, Szczecin,

Poland

\author{
Address for correspondence: \\ Ewa Stachowska, \\ Department of Biochemistry \\ and Human Nutrition, \\ Pomeranian Medical \\ University, \\ Broniewskiego 24 \\ 71-460 Szczecin, Poland \\ ewast@pum.edu.pl
}

Received: 07.08.2018

Accepted: 18.10.2018

\section{ABSTRACT}

Background \& Aims: Functional gastrointestinal disorders are prevalent worldwide and alterations of gutbrain axis and intestinal barrier integrity may play a pivotal role in both the pathophysiology and clinical course of these bowel malfunctions. We aimed to assess the prevalence of abdominal pain in a selected adult population of Poland to determine potential environmental factors associated with gastrointestinal complaints. Methods: There were 1479 individuals - 657 women (44.42\%) and 822 men (55.58\%), aged $24.20 \pm 6.08$ years. The responders fulfilled an authors' questionnaire based on Rome II and III criteria focused on the abdominal pain prevalence and environmental factors involved in its occurrence.

Results: The frequency of abdominal pain was found to be as high as $19.2 \%$. Male gender $(n=822)$ and basic education level $(\mathrm{n}=151)$ lowered the risk of abdominal pain occurrence $(\mathrm{OR}=0.7, \mathrm{p}<0.012$ and $\mathrm{OR}=0.5$, $\mathrm{p}<0.021$, respectively). Psychological distress, proton pump inhibitors (PPIs) and antibiotics usage were found as risk factors of abdominal pain $(\mathrm{OR}=2.503, \mathrm{p}<0.01 ; \mathrm{OR}=3.308, \mathrm{p}<0.01 ; \mathrm{OR}=3.105, \mathrm{p}<0.01$, respectively). Conclusions: Abdominal pain is prevalent in young adult inhabitants of Poland, especially in women. Intense psychological stress, as well as PPIs and antibiotics usage elevate the risk.

Key words: abdominal pain - defecation - stress - Rome criteria - Functional gastrointestinal disorders FGIDs - irritable bowel syndrome - functional dyspepsia.

Abbreviations: FD: functional dyspepsia; FGID: functional gastrointestinal disorders IBS: irritable bowel syndrome; NSAIDs: nonsteroidal anti-inflammatory drugs; PPI: proton pump inhibitors.

\section{INTRODUCTION}

Gastrointestinal complaints occur commonly in the general population with a growing number diagnosed with functional gastrointestinal disorders (FGIDs), which include irritable bowel syndrome (IBS), functional dyspepsia (FD), functional constipation, functional fecal incontinence, and functional anorectal pain [1]. The frequency, intensity, and type of these symptoms vary among individuals. The most frequent are bloating, indigestion, belching, flatulence and borborygmic [2]. Previous Rome II and III criteria included abdominal pain and discomfort in association with a change in the frequency or form of the stool. Abdominal discomfort was abandoned in the Rome IV revision. Functional anorectal pain syndromes are less frequent, inadequately studied and not necessarily associated with alteration in bowel habits. All these clinical entities have a high negative impact on the health related quality of life [3].

The number of adults affected by abdominal pain associated with altered bowel habits is substantial [4]. While it appears to be more prevalent among children and young adults, the effect of variables such as age remains uncertain. The prevalence of FGIDs varies between $10 \%$ to $20 \%$ in Western populations; most of the available studies are probably biased due to a heterogeneous patient selection methodology [5]. Currently, the Rome Foundation is performing a global epidemiological study surveying more than 70,000 individuals in more than 30 countries using the Rome IV diagnostic criteria, but the results and outcomes are still pending [6].

It is undeniable that FGIDs affect not only the patients, but also their families and employment, with significant detriment to their quality of life [7]. The severity of FGIDs is a main predictor of work and daily activity impairment as 
well as absenteeism from work and other activities, which result in substantial socioeconomic costs. It has been reported that yearly costs related to IBS care are at least comparable to costs devoted to all-cause health care, even after controlling for demographics and comorbidities [8]. Of relevance, it was shown that in FGID patients the incidence of depression and other neuropsychiatric disorders are elevated, implicating an increased economic burden [9]. It is therefore of the highest priority to improve diagnostic and treatment protocols for this large patient population.

To the best of our knowledge, no large-scale investigations on environmental factors related to FGIDs in healthy young people in Poland have been conducted. Therefore, this study assessed the presence of symptoms, and use of over the counter medication for suspected FGIDs in a population of young adults participating in a popular music festival in Poland.

\section{METHODS}

\section{Study population}

A cross-sectional survey in Kostrzyn, Poland, during the Woodstock Music Festival was carried out in 2016 [10]. Data was collected using electronic devices (tablets and smartphones) in which the questionnaire was installed as an application. Study subjects were randomly selected at main communication routes of the festival camp. Inclusion criteria was age between 18-35 years and no history of medically confirmed organic gastrointestinal disease. The surveyed group consisted of 1,484 persons, among them 822 men (55.58\%), aged $24.20 \pm 6.08$ years. There were 5 respondents who did not fit this age range; thus in total 1,479 questionnaires were analyzed.

The Medical Ethical Committee of the Pomeranian Medical University declared this a minimal risk protocol so as questionnaires were returned, they were stored anonymously with no patient identifiers. Written informed consent was not necessary, but oral consent was obtained at the time of patient recruitment to complete the survey.

\section{Questionnaire}

The questionnaire was specifically designed by the researchers to assess bowel habit, medication, and over the counter (OTC) supplementation use. The primary goal was to assess the presence of pain associated with suspected FGIDs which was assessed using the following questions: 1 . Have you been experiencing pain or unpleasant sensations in the abdomen for 12 months? 2. Have you experienced pain or unpleasant sensations in the abdomen during the last 6 months that disappeared after a bowel movement? 3. Have you experienced pain or unpleasant sensations in the abdomen which appeared with the change in the frequency of stool defecation? and 4 . Have you experienced pain or unpleasant sensations in the abdomen which appeared with the change of the consistency and appearance of stool?

The questions had to be answered with either "yes" or "no". The frequency of gastrointestinal symptoms was assessed on a three-point scale: $1=$ never, almost never, or once a month or less; 2 = several times a month or several times a week; $3=$ every day or several times a day.
The respondents were asked about the frequency of experiencing stress, usage of medications (nonsteroidal antiinflammatory drugs - NSAIDs, antibiotics, proton pump inhibitors - PPIs), consumption of selected food products (probiotics, alcohol), and having less than 7 hours of sleep. In this case we used a similar scale, ranging from 1 to 3 . These factors were further referred to as environmental factors. Selfreported factors such as degree of education and BMI were also recorded. We assumed that basic level of education was after primary school, secondary education was after high school, and high education during/after university studies.

\section{Statistics}

Statistical analyses were performed using $\mathrm{R}$ statistical software. Main variables were presented as either mean and standard deviations or number and percentage. The analysis was performed in relation to patients that reported one or more of the following symptoms:

- symptom pattern 1: pain or unpleasant sensations in the abdomen for 12 months ;

- symptom pattern 2: pain or unpleasant sensations in the abdomen during the last 6 months that disappeared after bowel movement;

- symptom pattern 3: pain or unpleasant sensations in the abdomen which appeared with the change in the frequency of stool defecation;

- symptom pattern 4: pain or unpleasant sensations in the abdomen which appeared with the change of the consistency and appearance of stool.

Having one or more of these symptoms was defined as having a problem with defecation. Logistic regression analysis was used to assess adjusted odds ratios in order to identify risk factors between the symptoms and environmental factors. Twosided $\mathrm{p}$ value $<0.01$ was considered as statistically significant.

\section{RESULTS}

The study included 1,479 individuals - 657 women (44.42\%) and 822 men (55.58\%), aged $24.20 \pm 6.08$ years. There were 151 people who declared basic education (10.21\%), 853 people with secondary education (57.67\%) and 475 people with higher education (32.12\%). The average BMI declared by the participants was $23.07 \pm 3.84 \mathrm{~kg} / \mathrm{m}^{2}$. Table I provides data on the number of participants reporting painful defecation.

The factors that reduced the risk of abdominal pain related to bowel habits were male sex $(n=822)$ and basic education level $(\mathrm{n}=151)(\mathrm{OR}=0.7, \mathrm{p}<0.012$ and $\mathrm{OR}=0.5, \mathrm{p}<0.021$, respectively).

The data also showed that stress was the main factor that increased the risk of abdominal pain and altered bowel habits. A direct relationship between level of stress and gastrointestinal complaints was noted. For example, patients who reported a stress frequency of 2 which equals stress several times a month or several times a week had a 1.5 times higher risk of gastrointestinal problems $(\mathrm{OR}=1.5 \mathrm{p}<0.01)$. When the declared frequency was 3 , meaning stress occurring every day or several times a day, the risk increased 2.5 times $(\mathrm{OR}=2.5, \mathrm{p}<0.01)$ (see Table II). However, we did not obtain data on what type of stress the participants were experiencing. We presume that in most of them was of mild, psychological origin. 
Table I. The number of subjects experiencing abdominal pain and disturbed defecation

\begin{tabular}{lcc}
\hline $\begin{array}{l}\text { Symptom patterns of } \\
\text { pain }\end{array}$ & $\begin{array}{c}\text { People declaring pain } \\
\mathrm{n}(\%)\end{array}$ & $\begin{array}{c}\text { People declaring no } \\
\text { pain } \mathrm{n}(\%)\end{array}$ \\
\hline Pattern 1 & $284(19.20)$ & $1195(80.80)$ \\
Pattern 2 & $185(12.51)$ & $1294(87.49)$ \\
Pattern 3 & $129(8.72)$ & $1350(91.28)$ \\
Pattern 4 & $135(9.13)$ & $1344(90.87)$ \\
\hline
\end{tabular}

n-number of subjects. For symptom pattern, please see the Methods chapter.

Results also showed that problems with defecation were significantly more common in women, and interestingly also in PPIs users. Depending on the frequency of medication administration, the risk increased 3.4 times (frequency=2; $\mathrm{p}<0.01)$. In the third category, the risk of abdominal pain and altered bowel habits increased 2.5 times $(\mathrm{p}<0.01)$. Similarly, the use of antibiotics increased the risk of pain occurrence 3 times (frequency $=2 ; \mathrm{p}<0.01$ ) and 1.4 times (frequency $=3 ; \mathrm{p}$ $<0.05)$. There was no relationship between the frequency of intake of NSAIDs and the occurrence of pain during bowel movement. However, we did not collect information on when the medication was taken. We assumed that the study participants considered the recent months, prior to the survey. Details are presented in Table II.

Table II. Multivariate Linear Regression of the factors affecting the abdominal pain related to bowel habits.

\begin{tabular}{lccc}
\hline Coefficient (95\% CI) & OR & $\begin{array}{c}\text { Response } \\
\text { n of population })\end{array}$ & p-value \\
\hline Male sex & 0.717 & $822(55.58)$ & 0.011 \\
Education (basic level) & 0.592 & $151(10.21)$ & 0.021 \\
Education (high level) & 0.919 & $475(55.58)$ & 0.527 \\
Stress frequency (2) & 1.521 & $705(47.67)$ & $<0.01$ \\
Stress frequency (3) & 2.503 & $220(14.87)$ & $<0.01$ \\
PPIs frequency (2) & 3.308 & $66(4.46)$ & $<0.01$ \\
PPIs frequency (3) & 2.577 & $16(1.08)$ & 0.081 \\
NSAIDs frequency (2) & 1.085 & $129(8.72)$ & 0.693 \\
NSAIDs frequency (3) & 0.695 & $23(1.56)$ & 0.469 \\
Antibiotic frequency (2) & 3.105 & $26(5.42)$ & $<0.01$ \\
Antibiotic frequency (3) & 1.472 & $15(1.01)$ & 0.0501 \\
\hline
\end{tabular}

CI: confidence interval; 2 = several times a month or several times a week; $3=$ every day or several times a day.

\section{DISCUSSION}

The aim of the present study was to expand the knowledge regarding the prevalence of abdominal pain and suspected FGIDs in a captive population of younger patients participating in a music festival and to look for environmental factors that could help identify risk factors implicated in the pathophysiology of suspected functional bowel disorders. In this population the incidence of abdominal pain varied between $8.72 \%$ to as much as $19.2 \%$. In contrast, Niemyjska et al. [11] evaluated IBS-related symptoms in students of Warsaw University and found that about $50 \%$ of study participants declared to suffer from abdominal pain at least once every week, depending on their residence and physical activity, thus related to the experience of psychological and physical stress. These results are however higher than those observed by Aziz et al. [4], who reported that the prevalence of symptom-based Rome IV FGIDs in adults from the United States, Canada and United Kingdom was approximately $10 \%$. Another survey of bowel habits in 2,273 children (mean age 13.2 years) established that abdominal pain related to defecation was present in $10.6 \%$ of them [12]. In a systematic review published later, it was shown that painful defecation among children was associated with stressful life events [13]. A single country study from Italy [14] indicated that severe IBS affected $23.5 \%$ of adults. Ziółkowski et al. [15] analyzed a population in a middlesize Polish city and found that $23 \%$ of participants suffered from dyspepsia and $36 \%$ had gastroesophageal reflux disease (GERD). Constipation was found to be present in $13 \%$ of participants, and bloating in $31 \%$. A minority of cases $(n=7)$ suffered from diarrhea. This indicates a wide variability of the prevalence of gastrointestinal symptoms indicative of FGIDs, some of which likely indicate methodological and patient population differences, so the results of this study also need to be considered cautiously.

The etiology of FGIDs is complex and not entirely elucidated. Rome Foundation listed several factors which contribute to FGIDs: i) motility disturbance, ii) visceral hypersensitivity, iii) altered mucosal and immune function; iv) altered gut microbiota, and v) altered central nervous system processing [16]. Identification of initial triggers of these abnormalities is an essential part of the initial patient assessment [17]. A few studies confirmed that intestinal barrier disruptions might be responsible for FGIDs onset and clinical prognosis. Experiments proved that both IBS and FD may be related to the imbalance within gut microbiota, tight junction dysregulation and gut associated lymphoid tissue (GALT) activation. Also, the evidence that multiple environmental factors might alter gut microbiota and thus gut-brain axis function is mounting [18-23]. In fact, a comprehensive psycho-neuro-immunological approach to abdominal pain management has been discussed [24]. However, the role of environmental factors in defecation-related abdominal pain in young people requires further research [25].

Our study points to the important role of stress in the occurrence of defecation-related abdominal pain. Stress plays an important role, and is a recognized risk factor for FGIDs [13]. This highlights the fact that despite numerous studies describing the potential negative effect of medication such as PPIs, antibiotics, and NSAIDs [26], the role of stress, its frequency and character are still being undervalued in everyday clinical practice also for FGIDs. The mechanisms linking psychological stress with intestinal inflammation are not fully understood, but studies indicate that stress increases the risk of intestinal inflammation in people and animals [27]. It can induce alterations of gastrointestinal microbiota, can lead to mucosal mast cell hyperplasia, and bacterial translocation across gut barrier, and hence gut inflammation [28]. In two recent cohort studies psychological distress significantly increased the risk of developing both IBS and FD emphasizing that bidirectional gut-brain axis neural and 
biochemical pathways may be disrupted by stress hormone release $[29,30]$. As in this study and other studies, females are at a greater risk of pain, and have a higher frequency of intestinal problems [31]. It is possible that there is a hormonal cause as sex hormone concentration during the menstrual cycle significantly affects sensitive-motor nerves within the digestive tract, and also modulates sensitivity to stress via gut barrier disruptions, thus altering the gut brain axis function [32].

Interestingly, in the present study, a reduction of the risk of defecation-related abdominal pain among people with a lower level of education was found. It is possible that this is in part due to a lesser exposure of this group to work-related stress. However, we collected no data on profession, which might introduce a potential bias into these results. Similar results were observed in individuals diagnosed with inflammatory bowel disease (IBD), where work-related stress was measured with the aid of a special questionnaire and scores. As shown in the study, overcommitment scores were higher in relation to full-time employees with a high level of education [33]. These data also add to the evidence of the importance of gut-brain communication and its role in the development of gastrointestinal symptoms [34].

Beside stress, other environmental factors, such as PPIs and antibiotics were found to be significant triggers, capable of generating FGIDs symptoms. PPIs but not NSAIDs may decrease $a$ diversity in the gut microbiome. It was found that PPIs lower the abundance of Clotridiales and increase the copy numbers of Actinomycetales, Micrococcaceae and Streptococcaceae, all increasing susceptibility to Clostridium difficile infection, risk of visceral hypersensitivity and occurrence of FGIDs related symptoms [35].

Our study has both strengths and limitations. A heterogeneous patient population from different parts of Poland was included, thus the sample may be considered as representative especially for this age group. The questionnaire was author-developed and, while based on the Rome III criteria, would require validation. It would be interesting to apply the same methodology using the Rome IV criteria [36]. While this study did not capture the frequency of symptom recurrence, it did capture the presence of symptoms in the last 12 months. Currently there is a trend to simplify the diagnostic criteria [6], as many physicians do not recognize them in their professional practice [37].

\section{CONCLUSION}

The results of this app-based electronic poll indicate that gastrointestinal symptoms are common in young adults in Poland. The data justify undertaking large-scale epidemiological studies among young people in Poland with the aim to investigate the problem of stress-related chronic abdominal symptoms. Further attention should be focused on the stress as one of the main factors negatively influencing public health, as it has potentially significant long-term economical and social impacts.

Conflicts of interest: None to declare.
Authors' contributions: E.S: first draft of the manuscript; E.S., K.S.Z. and W.M.: manuscript writing; P.B.: statistical analysis. All the authors collected data and approved the final manuscript.

Acknowledgements: We thank Mr Mateusz Sawinda for his technical support and all the attendants of Woodstock Festival 2016 for their agreement to participate in our study.

We acknowledge Prof. Luis F. Lara from the Ohio State University, Columbus, Ohio, USA for reviewing the paper and for expert English proof reading.

Funding: This study did not receive any specific grant from funding agencies in the public, commercial, or not-for-profit sectors.

\section{REFERENCES}

1. Samiullah S, Malone M, Waheed A. Functional Gastrointestinal Disorders: Approach to Patients With Functional Gastrointestinal Disorders. FP Essent 2018;466:11-13.

2. Azpiroz F, Malagelada JR. Abdominal bloating. Gastroenterology 2005;129:1060-1078. doi:10.1053/j.gastro.2005.06.062

3. Malone M, Waheed A, Samiullah S. Functional Gastrointestinal Disorders: Functional Lower Gastrointestinal Disorders in Adults. FP Essent 2018;466:21-28.

4. Aziz I, Palsson OS, Törnblom H, Sperber AD, Whitehead WE, Simrén M. The Prevalence and Impact of Overlapping Rome IV-Diagnosed Functional Gastrointestinal Disorders on Somatization, Quality of Life, and Healthcare Utilization: A Cross-Sectional General Population Study in Three Countries. Am J Gastroenterol 2018;113:86-96. doi:10.1038/ ajg.2017.421

5. Sperber AD, Dumitrascu D, Fukudo S, et al. The global prevalence of IBS in adults remains elusive due to the heterogeneity of studies: a Rome Foundation working team literature review. Gut 2017;66:1075-1082. doi:10.1136/gutjnl-2015-311240

6. Sood R, Camilleri M, Gracie DJ, et al. Enhancing Diagnostic Performance of Symptom-Based Criteria for Irritable Bowel Syndrome by Additional History and Limited Diagnostic Evaluation. Am J Gastroenterol 2016;111:1446-1454. doi:10.1038/ajg.2016.308

7. Corsetti M, Whorwell P. The global impact of IBS: time to think about IBS-specific models of care? Therap Adv Gastroenterol. 2017;10:727736. doi:10.1177/1756283X17718677

8. Buono JL, Mathur K, Averitt AJ, Andrae DA. Economic Burden of Irritable Bowel Syndrome with Diarrhea: Retrospective Analysis of a U.S. Commercially Insured Population. J Manag Care Spec Pharm 2017;23:453-460. doi:10.18553/jmcp.2016.16138

9. Fadgyas-Stanculete M, Buga AM, Popa-Wagner A, Dumitrascu DL. The relationship between irritable bowel syndrome and psychiatric disorders: from molecular changes to clinical manifestations. J Mol Psychiatry 2014;2:4. doi:10.1186/2049-9256-2-4

10. Woodstock 2016: Szczegółowy program koncertów - gazetalubuska.pl [Internet]. [cited 2018 Oct 16]. Available from: https://gazetalubuska. pl/woodstock-2016-szczegolowy-program-koncertow/ar/10064502

11. Niemyjska S, Ukleja A, Ławiński M. Evaluation Of Irritable Bowel Syndrome Symptoms Amongst Warsaw University Students. Pol Przegl Chir 2015;87:252-259. doi: 10.1515/pjs-2015-0050

12. Devanarayana NM, Rajindrajith $\mathrm{S}$. Bowel habits and behaviors related to defecation in 10- to 16-year-olds: impact of socioeconomic 
characteristics and emotional stress. J Pediatr Gastroenterol Nutr 2011;52:569-573. doi:10.1097/MPG.0b013e3181fd082b

13. Qin HY, Cheng CW, Tang XD, Bian ZX. Impact of psychological stress on irritable bowel syndrome. World J Gastroenterol 2014;20:141261431. doi:10.3748/wjg.v20.i39.14126

14. Manzoli L, Flacco ME, Marzuillo C, Lopetuso L. Prevalence of severe irritable bowel syndrome among Italian adults. A meta-analysis. Eur Rev Med Pharmacol Sci 2017;21:5751-5764. doi:10.26355/ eurrev_201712_14022

15. Ziółkowski BA, Pacholec A, Kudlicka M, Ehrmann A, Muszyński J. Prevalence of abdominal symptoms in the Polish population. Gastroenterology Rev 2012;7:20-25. doi:10.5114/pg.2012.27218

16. Vork L, Keszthelyi D, Mujagic Z, et al. Development, content validity, and cross-cultural adaptation of a patient-reported outcome measure for real-time symptom assessment in irritable bowel syndrome. Neurogastroenterol Motil 2018;30(3). doi:10.1111/nmo.13244

17. Moayyedi P, Ford AC, Talley NJ, et al. The efficacy of probiotics in the treatment of irritable bowel syndrome: a systematic review. Gut 2010;59:325-332. doi:10.1136/gut.2008.167270

18. Farup PG, Ueland T, Rudi K, Lydersen S, Hestad K. Functional Bowel Disorders Are Associated with a Central Immune Activation. Gastroenterol Res Pract 2017;2017:1642912. doi:10.1155/2017/1642912

19. Marlicz W, Yung DE, Skonieczna-Żydecka K, et al. From clinical uncertainties to precision medicine: the emerging role of the gut barrier and microbiome in small bowel functional diseases. Expert Rev Gastroenterol Hepatol 2017;11:961-978. doi:10.1080/17474124.2017.1343664

20. Zhong L, Shanahan ER, Raj A, et al. Dyspepsia and the microbiome: time to focus on the small intestine. Gut 2017;66:1168-1169. doi:10.1136/ gutjnl-2016-312574

21. Mujagic Z, de Vos P, Boekschoten MV, et al. The effects of Lactobacillus plantarum on small intestinal barrier function and mucosal gene transcription; a randomized double-blind placebo controlled trial. Sci Rep 2017;7:40128. doi:10.1038/srep40128

22. Tan VP, Liu KS, Lam FY, Hung IF, Yuen MF, Leung WK. Randomised clinical trial: rifaximin versus placebo for the treatment of functional dyspepsia. Aliment Pharmacol Ther 2017;45:767-776. doi:10.1111/ apt.13945

23. Giamarellos-Bourboulis E, Tang J, Pyleris E, et al. Molecular assessment of differences in the duodenal microbiome in subjects with irritable bowel syndrome. Scand J Gastroenterol 2015;50:1076-1087. doi:10.31 09/00365521.2015.1027261

24. Toljan K, Vrooman B. Psychoneuroimmunological approach to gastrointestinal related pain. Scand J Pain 2017;17:431-443.
25. Holtmann G, Shah A, Morrison M. Pathophysiology of Functional Gastrointestinal Disorders: A Holistic Overview. Dig Dis 2017;35 Suppl 1:5-13. doi:10.1159/000485409

26. Sood R, Ford AC. Diagnosis: Rome IV criteria for FGIDs - an improvement or more of the same? Nat Rev Gastroenterol Hepatol 2016;13:501-502. doi:10.1038/nrgastro.2016.110

27. Gao X, Cao Q, Cheng Y, et al. Chronic stress promotes colitis by disturbing the gut microbiota and triggering immune system response. Proc Natl Acad Sci U S A 2018;115:E2960-E2969. doi:10.1073/ pnas. 1720696115

28. Cui G, Yuan A. A Systematic Review of Epidemiology and Risk Factors Associated With Chinese Inflammatory Bowel Disease. Front Med (Lausanne) 2018;5:183. doi:10.3389/fmed.2018.00183

29. Jones MP, Tack J, Van Oudenhove L, et al. Mood and Anxiety Disorders Precede Development of Functional Gastrointestinal Disorders in Patients but Not in the Population. Clin Gastroenterol Hepatol 2017;15:1014-1020.e4. doi:10.1016/j.cgh.2016.12.032

30. Koloski NA, Jones M, Talley NJ. Evidence that independent gut-to-brain and brain-to-gut pathways operate in the irritable bowel syndrome and functional dyspepsia: a 1-year population-based prospective study. Aliment Pharmacol Ther 2016;44:592-600. doi:10.1111/apt.13738

31. Adeyemo MA, Spiegel BM, Chang L. Meta-analysis: do irritable bowel syndrome symptoms vary between men and women? Aliment Pharmacol Ther 2010;32:738-755. doi:10.1111/j.13652036.2010.04409.x

32. Meleine M, Matricon J. Gender-related differences in irritable bowel syndrome: Potential mechanisms of sex hormones. World J Gastroenterol 2014;20:6725-6743. doi:10.3748/wjg.v20.i22.6725

33. Schreiner P, Biedermann L, Rossel JB, et al. Prevalence and Determinants of Job Stress in Patients with Inflammatory Bowel Disease. Inflamm Bowel Dis 2017;23:310-317. doi:10.1097/MIB.0000000000001012

34. Quigley EMM. The Gut-Brain Axis and the Microbiome: Clues to Pathophysiology and Opportunities for Novel Management Strategies in Irritable Bowel Syndrome (IBS). J Clin Med 2018;7:6. doi:10.3390/ jcm7010006

35. Enck P, Mazurak N. Dysbiosis in Functional Bowel Disorders. Ann Nutr Metab 2018;72:296-306. doi:10.1159/000488773

36. Lacy BE, Mearin F, Chang L, et al. Bowel Disorders. Gastroenterology 2016;150:1393-1407.e5. doi:10.1053/j.gastro.2016.02.031

37. Mujagic Z, Jonkers DMAE, Hungin APS, et al. Use of Rome criteria for the diagnosis of irritable bowel syndrome in primary care: a survey among European countries. Eur J Gastroenterol Hepatol 2017;29:651656. doi:10.1097/MEG.0000000000000848 